\title{
Parotid Gland Oncocytoma: A Rare Case and Literature Review
}

\author{
Amel El Korbia, b, c, e, Sondes Jellalia, b, Manel Njima ${ }^{\text {a, d }}$, Khaled Harrathia, b, \\ Rachida Bouatay $^{\mathrm{a}, \mathrm{b}}$, Mehdi Ferjaoui ${ }^{\mathrm{a}, \mathrm{b}}$, Naourez Kolsi ${ }^{\mathrm{a}, \mathrm{b}}$, c, \\ Jamel Koubaa ${ }^{a, b}$
}

\begin{abstract}
Oncocytoma is a rare salivary gland tumor consisting of oncocytes with many hyperplastic mitochondria. It usually occurs in the parotid gland. Clinical diagnosis is often challenging due to the likeness of oncocytoma to other benign and low-grade malignant salivary gland tumors. We report a case of a patient who presented to our department with a right parotid swelling evolving for 1 year. Imaging studies diagnosed the lesion as a benign tumor. The patient underwent superficial parotidectomy with complete tumor excision with no complication. Definitive histology examination concludes to oncocytoma. The patient is disease free with a follow-up of 3 years.
\end{abstract}

Keywords: Oncocytoma; Parotid; Magnetic resonance imaging; Surgery

\section{Introduction}

Oncocytomas are rare tumors and represent less than $1 \%$ of all salivary gland tumors [1]. Most oncocytomas arise in the parotid gland. They are considered as benign tumors and usually present as a solitary mass. Diagnosis is suggested by imaging. Diagnostic confirmation is histological after parotidectomy $[1,2]$.

\section{Case Report}

A 62-year-old man, presented to our department with a history

Manuscript submitted March 27, 2019, accepted April 12, 2019

aUniversity of Monastir, Monastir, Tunisia

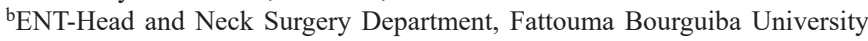
Hospital of Monastir, Monastir, Tunisia

cHealthcare Research Unit, UP12SP41, Tunisia

dPathologic Anatomy and Cytology Department, Fattouma Bourguiba Hospital of Monastir, Monastir, Tunisia

e Corresponding Author: Amel El Korbi, ENT Department, Fattouma Bourguiba Hospital of Monastir, University of Monastir, Healthcare Research Unit UR12SP41, Tunisia. Email: elkorbiamel@yahoo.fr

doi: https://doi.org/10.14740/jmc3288 of painless mass at the level of the right parotid region with a progressive growth evolving for 1 year. Physical examination revealed a $20 \mathrm{~mm}$ well-defined mass of right parotid gland. There were no palpable cervical lymph nodes and no facial paralysis. The Stenon's canal orifice was free at the intraoral exam. Ultrasound exam showed a well circumscribed a mass (20 $\mathrm{mm}$ in diameter) of superficial lobe of the right parotid gland. This lesion was hypoechoic and vascularized (Fig. 1).

Magnetic resonance imaging (MRI) showed a hyposignal lesion on T1-weighted imaging of the superficial portion of the right parotid gland with homogenous enhancement after gadolinium. The mass was slight hypersignal on T2-weighted imaging. Axial diffusion-weighted imaging found a hypersignal with an apparent diffusion coefficient (ADC) of 1.36 (Fig. 2). Based on MRI findings, the diagnosis of benign tumor was made.

The patient underwent superficial parotidectomy with complete tumor excision and facial nerve preservation. Frozen section examination was not contributive. We decided not to complete parotid excision and waited for definitive histology. No complications were observed after surgery, particularly no facial palsy nerve.

Microscopic analysis revealed a well-limited benign cells proliferation surrounded by a thin fibrous capsule. It was made by sheets or trabeculae, patterns of monotonous large polygonal cells with well-defined cell borders, deeply eosinophilic

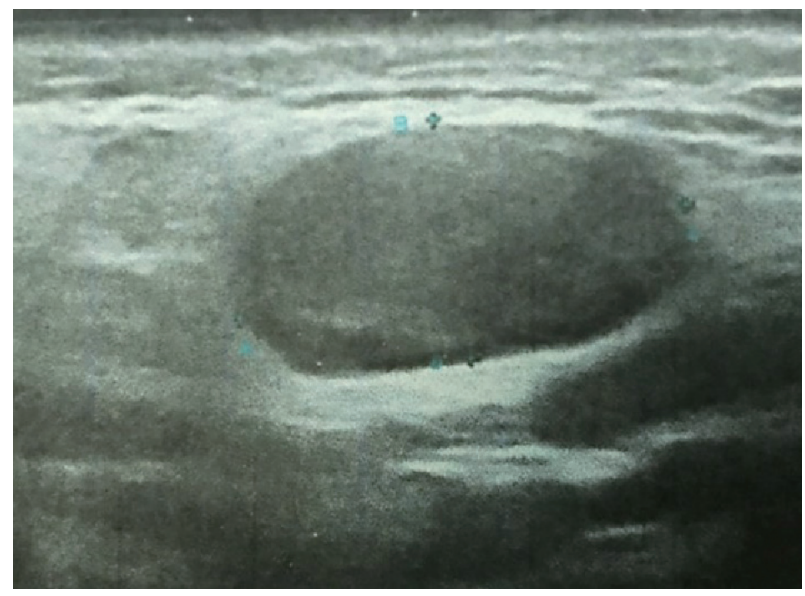

Figure 1. Parotid ultrasonography shows a $20 \mathrm{~mm}$ in diameter wellcircumscribed mass of the superficial lobe of the right parotid gland. The mass is hypoechoic. 


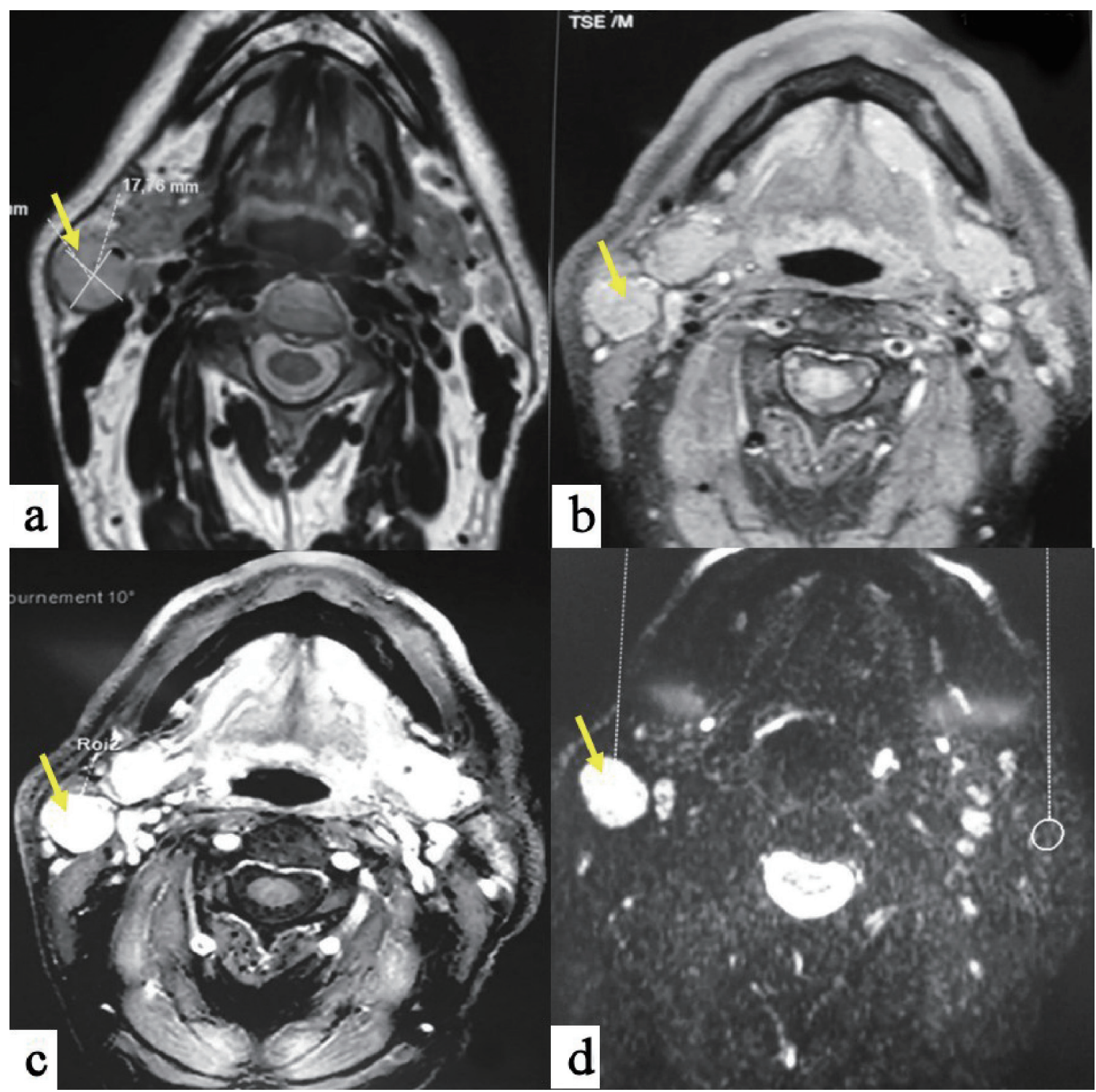

Figure 2. MRI in T1-weighted sequences (a) shows a hyposignal mass of the superficial lobe of the right parotid gland; with homogenous enhancement after gadolinium injection (b). The mass is slight hypersignal on T2-weighted sequences (c), and with a hypersignal on diffusion sequences (d).

and granular cytoplasm. Nuclei were monomorphic and finely nucleated. There was neither mitosis nor necrosis. At the same time connective tissue was fibrous and edematous with many vessels (Fig. 3). These findings confirmed the diagnosis of parotid oncocytoma. We observed no recurrence after 3 years of follow-up.

\section{Discussion}

The word "oncocyte" was first introduced in 1931 by Hamperl [1]. Oncocytomas are rare tumors that account for $0.1-1.5 \%$ of salivary gland tumors and $2.3 \%$ of benign epithelial salivary gland neoplasms [1-3]. Oncocytomas were first described by Jaffe in 1932 [2]. The parotid is the most commonly involved gland accounting for $78-84 \%$ of salivary gland oncocytomas [2]. Oncocytomas are benign epithelial tumors characterized by oncocytes with eosinophilic granular cytoplasm that contains mitochondria. Oncocytic cells are thought to originate from the transformation of epithelial cells of salivary gland ducts or acini [4]. They occur most commonly in the sixth to eighth decades and are slightly predominant in female gender [2].

Although malignant transformation of this tumor is unusual, clinical follow-up is important because malignant oncocytoma may not be correctly diagnosed owing to histological similarities with benign oncocytoma [5].

Pathogenesis is not exactly determined, although mitochondrial functional lacks are believed to induce progressive degeneration of the epithelial salivary cells. In the literature, we report that only one mitochondrial DNA rearrangement 


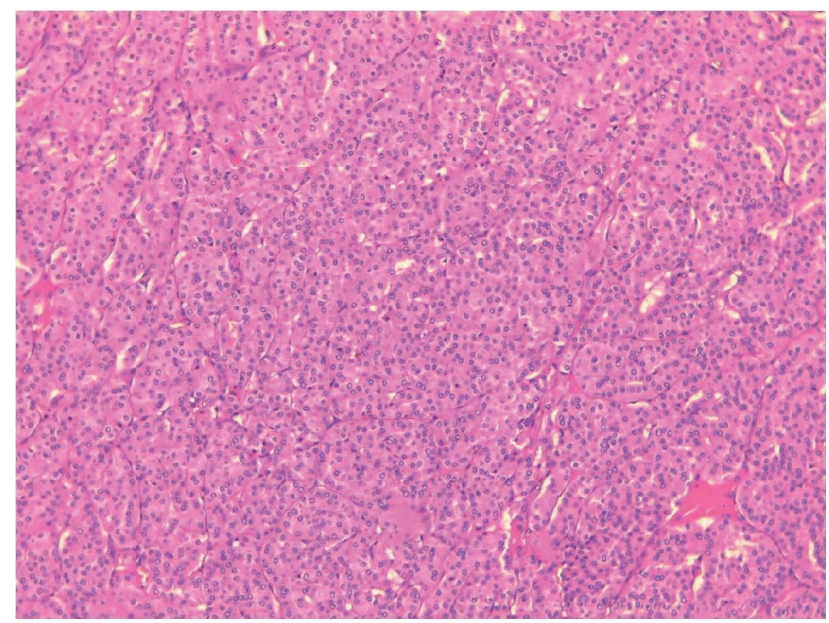

Figure 3. Sheets or trabeculae, patterns of monotonous large polygonal cells with well defined cell borders, deeply eosinophilic and granular cytoplasm (H\&E, × 100).

(among 200 described cases) has been in relation with parotid tumor genesis. The implication of some viruses, such as Epstein-Barr virus (EBV), human immunodeficiency virus (HIV), human herpes virus-8 (HHV-8), human T lymphotropic virus type 1 (HTLV-1) and human papillomavirus (HPV) with parotid neoplasms has been documented [6].

Clinical presentation of oncocytomas is similar to other benign salivary tumors with no specificity. They present as lobulated and mobile mass [3]. Bilateralism is observed in $7 \%$ of cases [7]. In the literature, we report a case of facial palsy associated to parotid oncocytoma, which was explained by ischemia and strangulation of the nerve secondary to tumoral inflammation [1]. The newest World Health Organization (WHO) classification describes three entities: nodular oncocytic hyperplasia, oncocytoma, and oncocytic carcinoma [8]. Oncocytic changes of secretory epithelia are thought to be metaplastic, a protective phenomenon against adverse change. Aging is also thought to cause a functional exhaustion of the mitochondrial enzymes and a compensatory mitochondrial hyperplasia to overcome an energy-deficient condition [9]. Oncocytic lesions of the parotid gland demonstrate a spectrum of changes ranging from oncocytosis to benign oncocytomas and oncocytic carcinomas. There have been reports of the coexistence of these oncocytic entities. This raises the concept of a progression model with a progressive transition between these oncocytic lesions [9].

The ultrasound features of parotid oncocytomas are not specific and include a hypoechoic mass with well-defined margins, like other benign parotid tumors such as pleomorphic adenomas [10].

Due to their low prevalence, only few case reports on the MRI features of parotid oncocytomas are available in the published literature $[3,5]$. The reports on MRI of parotid oncocytomas describe these tumors as demonstrating T1 and T2 hyposignal with homogeneous contrast enhancement. The decreased signal intensity on both T1- and T2-weighted images is explained by high cellularity and low free water content of oncocytoma [4].

In the literature, MRI characteristic of parotid oncocytoma is well-defined parotid mass with homogeneous enhancement. The important differential diagnoses for well-defined enhancing parotid tumors seen on MRI include the Warthin's tumor and basal cell adenomas [4].

Diagnosis by fine-needle aspiration cytology (FNAC) may be very difficult. In fact, we observe oncocytic changes in a large variety of neoplastic and non-neoplastic conditions; otherwise focal sampling of the lesion explains cytology misdiagnosis [1]. It has been reported the sensitivity for the detection of oncocytoma by FNAC is $29 \%$ [11].

Complete surgical excision is the gold standard of treatment. The choice of total or superficial parotidectomy is predicted preoperatively by clinico-radiological findings, and preoperative tumor extensions. There is no need for adjuvant treatment such us chemotherapy and/or radiotherapy because of benign nature and slow growth rate of the tumor [12]. Nevertheless, we recommend long-term follow-up because some cases of malignant oncocytoma have been reported in the literature [13].

Recurrences are reported in less than $20 \%$ of cases, explained by incomplete surgical resection [12].

\section{Conclusions}

Parotid oncocytoma is benign epithelial tumor that frequently occurs between the sixth and the eighth decades of life. Clinical presentation is not specific with a parotid gland swelling and solid solitary mass on palpation. Oncocytoma should be assessed using MRI studies to evaluate the extending of the tumor. Upon histological verification, a surgical approach should be considered to eradicate the tumor and remove the parotid gland.

\section{Acknowledgments}

Not applicable.

\section{Financial Disclosure}

None to declare.

\section{Conflict of Interest}

None to declare.

\section{Informed Consent}

Patient's informed consent for publication of this report was obtained.

\section{Author Contributions}

Amel El Korbi: bibliography and writing the manuscript; 
Sondes Jellali: bibliography and writing the manuscript; Manel Njima: preparation of figures and comments; Khaled Harrathi: revision of the manuscript; Rachida Bouatay: bibliography; Mehdi Ferjaoui: bibliography; Naourez Kolsi: bibliography ; Jamel Koubaa: revision of the manuscript.

\section{References}

1. Hamada S, Fujiwara K, Hatakeyama H, Homma A. Oncocytoma of the parotid gland with facial nerve paralysis. Case Rep Otolaryngol. 2018;2018:7687951.

2. Shellenberger TD, Williams MD, Clayman GL, Kumar AJ. Parotid gland oncocytosis: CT findings with histopathologic correlation. AJNR Am J Neuroradiol. 2008;29(4):734-736.

3. Tan TJ, Tan TY. CT features of parotid gland oncocytomas: a study of 10 cases and literature review. AJNR Am J Neuroradiol. 2010;31(8):1413-1417.

4. Kasai T, Motoori K, Hanazawa T, Nagai Y, Ito H. MR imaging of multinodular bilateral oncocytoma of the parotid gland. Eur J Radiol Extra. 2007;63:97-100.

5. Sakai E, Yoda T, Shimamoto H, Hirano Y, Kusama M, Enomoto S. Pathologic and imaging findings of an oncocytoma in the deep lobe of the left parotid gland. Int J Oral Maxillofac Surg. 2003;32(5):563-565.

6. Vlachaki E, Tsapas A, Dimitrakopoulos K, Kontzoglou
G, Klonizakis I. Parotid gland oncocytoma: a case report. Cases J. 2009;2:6423.

7. Stomeo F, Meloni F, Bozzo C, Fois V, Pastore A. Bilateral oncocytoma of the parotid gland. Acta Otolaryngol. 2006;126(3):324-326.

8. Seethala RR, Stenman G. Update from the 4th Edition of the World Health Organization Classification of head and neck tumours: tumors of the salivary Gland. Head Neck Pathol. 2017;11(1):55-67.

9. Prabakaran SS, Chen F, Aguirre A. Oncocytoma of the parotid gland and its mimickers: a comprehensive review. N Am J Med Sci. 2010;3(4):171-180.

10. Lee YY, Wong KT, King AD, Ahuja AT. Imaging of salivary gland tumours. Eur J Radiol. 2008;66(3):419436.

11. Capone RB, Ha PK, Westra WH, Pilkington TM, Sciubba JJ, Koch WM, Cummings CW. Oncocytic neoplasms of the parotid gland: a 16-year institutional review. Otolaryngol Head Neck Surg. 2002;126(6):657-662.

12. Sepulveda I, Platin E, Spencer ML, Mucientes P, Frelinghuysen M, Ortega P, Ulloa D. Oncocytoma of the parotid gland: a case report and review of the literature. Case Rep Oncol. 2014;7(1):109-116.

13. Giordano G, Gabrielli M, Gnetti L, Ferri T. Oncocytic carcinoma of parotid gland: a case report with clinical, immunohistochemical and ultrastructural features. World J Surg Oncol. 2006;4:54 